\title{
ACADEMIA
}

Accelerating the world's research.

\section{Comparison of pap smear, visual inspection with acetic acid, human papillomavirus DNA-PCR testing and cervicography}

Eric Njiru

International Journal of Gynecology \& Obstetrics

\section{Cite this paper}

Downloaded from Academia.edu $₫$

Get the citation in MLA, APA, or Chicago styles

\section{Related papers}

Performance of the Roche AMPLICOR human papillomavirus (HPV) test in prediction of cervic... Joseph Monsonego

The value of visual inspection with acet ic acid and Pap smear in cervical cancer screening program in ... Vu Quoc Huy Nguyen, Le Tam

Risk est imates for persist ent high-risk human papillomavirus infect ions as surrogate endpoints of pr...

S. Syrjänen, Luis Sarian, S. Tatti 


\title{
Comparison of pap smear, visual inspection with acetic acid, human papillomavirus DNA-PCR testing and cervicography
}

\author{
H. De Vuyst ${ }^{a}$, P. Claeys ${ }^{a}$, S. Njirub ${ }^{b}$, L. Muchiri ${ }^{c}$, S. Steyaert ${ }^{d}$, P. De Sutter ${ }^{e}$, \\ E. Van Marck ${ }^{f}$, J. Bwayo ${ }^{b}$, M. Temmerman ${ }^{a, *}$
}

anternational Centre for Reproductive Health, Ghent University, De Pintelaan, 185, B-9000 Ghent, Belgium ${ }^{\mathrm{b}}$ Department of Medical Microbiology, University of Nairobi, Nairobi, Kenya

${ }^{\mathrm{C}}$ Department of Human Pathology, University of Nairobi, Nairobi, Kenya

${ }^{\mathrm{d} D e p a r t m e n t}$ of Clinical Biology, Microbiology and Immunology, Ghent University Hospital, Ghent, Belgium ${ }^{\mathrm{e}}$ Department of Gynecology and Obstetrics, Gynecological Oncology Unit and Colposcopy Clinic, Free University of Brussels, Brussels, Belgium

${ }^{\mathrm{f}}$ Department of Pathology, Antwerp University, Antwerp, Belgium

Received 3 August 2004; received in revised form 12 January 2005; accepted 12 January 2005

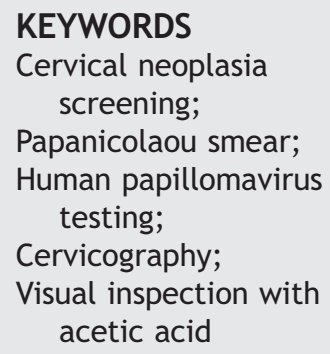

\begin{abstract}
Objective: To assess the test qualities of four screening methods to detect cervical intra-epithelial neoplasia in an urban African setting. Method: Six hundred fiftythree women, attending a family planning clinic in Nairobi (Kenya), underwent four concurrent screening methods: pap smear, visual inspection with acetic acid (VIA), PCR for high risk human papillomavirus (HR HPV) and cervicography. The presence of cervical intra-epithelial neoplasia (CIN) was verified by colposcopy or biopsy. Result: Sensitivity (for CIN2 or higher) and specificity (to exclude any CIN or cancer) were $83.3 \%(95 \% \mathrm{Cl}[73.6,93.0])$ and $94.6 \%(95 \% \mathrm{Cl}[92.6,96.5])$, respectively, for pap smear; $73.3 \%(95 \% \mathrm{Cl}[61.8,84.9])$ and $80.0 \%(95 \% \mathrm{Cl}[76.6,83.4])$ for VIA; $94.4 \%(95 \% \mathrm{Cl}[84.6,98.8])$ and $73.9 \%(95 \% \mathrm{Cl}[69.7,78.2])$ for HR HPV; and $72.3 \%(95 \% \mathrm{Cl}[59.1,85.6])$ and $93.2 \%(95 \% \mathrm{Cl}[90.8,95.7])$ for cervicography. Conclusion: The pap smear had the highest specificity (94.6\%) and HPV testing
\end{abstract}

* Corresponding author. Fax: +32 92403867 .

E-mail address: marleen.temmerman@ugent.be (M. Temmerman).

0020-7292/\$ - see front matter (c) 2005 International Federation of Gynecology and Obstetrics. Published by Elsevier Ireland Ltd. All rights reserved.

doi:10.1016/j.ijgo.2005.01.035 
the highest sensitivity (94.4\%). The visual methods, VIA and cervicography, were similar and showed an accuracy in between the former two tests.

(c) 2005 International Federation of Gynecology and Obstetrics. Published by Elsevier Ireland Ltd. All rights reserved.

\section{Introduction}

Screening for cervical lesions has proven successful in the industrialized world, with incidences of cervical cancer reduced by $80 \%$ in countries with organized screening and treatment programs [1]. The success of these programs can largely be attributed to the use of the Papanicolaou (pap) smear [2]. In the developing world, however, the incidence of cervical cancer remains high, particularly in sub-Saharan Africa, where cervical cancer is the most common cancer in women [3]. The lack of organized cervical cancer control programs is the main reason for this. Effective cytological screening programs are difficult to implement in low resource settings because of high cost, training requirements for laboratory technicians, and competing health priorities. Alternative screening methods are therefore being assessed. This paper reports on the test qualities of pap smear, visual inspection with acetic acid (VIA), cervicography, and DNA-PCR testing for human papillomavirus (HPV).

\section{Materials and methods}

Between January 1998 and July 2000, a crosssectional study was carried out in non-pregnant women aged 25-55 attending a family planning clinic in Nairobi, Kenya. The study was approved by the National Ethical Review Committee of Kenyatta National Hospital, Nairobi. The staff consisted of a study nurse, who received a 4-day training in the technique of VIA with a pictorial atlas for visual inspection of the cervix [4] and projected images of cervices, followed by hands-on training, and two medical doctors trained in colposcopy.

Each working day, the first eight consecutive clients presenting at the family planning clinic were invited to participate in the study. A signed informed consent was obtained from all participants. The study nurse was blinded to the clinical background (referred patients or women attending for family planning). At the first visit (V1), exfoliative cervical cells were obtained using a Cervex Brush (Rovers Medical Devices, Oss, The Netherlands). One smear was made on a glass slide for staining according to the Papanicoloau method. The brush was then submerged and stirred in $10 \mathrm{ml}$ phosphate buffered saline (PBS) and frozen at $-20{ }^{\circ} \mathrm{C}$. A VIA (VIA1) examination was then performed (inspecting the transformation zone) after application of commercially available vinegar (acetic acid 3-5\%) for $2 \mathrm{~min}$ and illumination with a halogen torch. VIA findings were recorded by the study nurse in a clinical records form (CRF). Two photographic slides were taken with a specially designed $35 \mathrm{~mm}$ reflex camera (cervicography) (National Testing Laboratories, Fenton, USA). Serum samples were obtained for HIV testing after pretest counseling. Blood samples were tested for HIV-1 and HIV-2 using ELISA Detect HIV1/2 (Immunosystems, Montreal, Quebec) and Recombigen HIV1/2 (Trinity Biotech, Galway, Ireland). All the women were invited to come for a follow-up visit after 3 weeks (V2). At this visit, VIA (VIA2) was performed on all women by the study nurse and colposcopy by the doctor. After these examinations, the findings were registered in the CRF and the result of the pap smear disclosed to the examinators and communicated to the woman. If the pap smear, VIA2, or colposcopy was positive, a biopsy and/or endocervical curettage was obtained.

Pap smears and cervical biopsies were processed and analyzed at the Department of Human Pathology, University of Nairobi, Kenya. Cytology was reported according to the Bethesda 1991 classification. All positive smears and $60 \%$ of the negatives, randomly chosen, were sent to the Department of Pathology, Antwerp University, Belgium for quality review. A Cohen's Kappa for agreement of 0.623 was found between the two institutions. The original Nairobi results before quality review were used in this analysis. All histology specimens were also reviewed and the Antwerp results were used for final data analysis. HPV samples were shipped to the Laboratory of Virology, Ghent University, Belgium for HPV DNA extraction, detection and genotyping, as described earlier [5]. Cervicography slides were assessed at the department of gynecology, Free University of Brussels, Belgium, by a senior staff colposcopist and accredited cervicography evaluator (PDS).

All examiners interpreting pap smears, HPV DNA $\mathrm{PCR}$, or cervicographies were blinded to the clinical background and to other screening test results. 
A pap smear was considered positive in case of LSIL or more severe lesions. VIA was considered positive in case of well defined, distinct acetowhite lesions on the cervix, close to the os. Colposcopy was considered positive for a colposcopic impression of LSIL or more. Any abnormal result on biopsy was taken as the final positive diagnosis, hereafter called the "reference test". If biopsy was negative, or in case there was no indication for biopsy (all screening tests and colposcopy were negative), the reference test was considered negative. Lesions of CIN2 or worse on the reference test are considered as diseased and reported as 'cases'. The presence of any HPV DNA resulted in a positive HPV test, but we also assessed the presence of DNA from high-risk HPV types (HPV HR).

Cervicographies were reported using the classification criteria proposed by Schneider et al. [6]. All grades equal to or higher than a low grade lesion were considered as a positive test result.

Data were entered in Epi-info (CDC, USA; WHO, Geneva) and analyzed in SPSS 10.0.5 for windows (SPSS, Chicago, IL, USA). Comparisons of categorical variables were made using Pearson's X2 and Fisher's exact tests. Means of continuous variables were compared using the Independent Samples $t$ test. Exact 95\% confidence intervals for diagnostic sensitivity (positive test results compared to the reference test) and specificity (negative test results compared to the reference test) were reported where appropriate.

\section{Results}

\subsection{Characteristics of the study population}

Of the 816 women presenting at Visit 1, 653 attended the clinic for visit 2 and were included in the study. The majority $(548,83.9 \%)$ were women who attended the clinic for family planning

Table 1 Comparison of risk factors for cervical (pre-) cancer between the screening and referred group

\begin{tabular}{lccc}
\hline & $\begin{array}{l}\text { Screening } \\
(N=548)\end{array}$ & $\begin{array}{l}\text { Referred } \\
(N=105)\end{array}$ & $p$ \\
\hline Mean age & 35.8 & 34.1 & 0.02 \\
Mean parity & 2.9 & 2.7 & 0.4 \\
Mean age first sex & 19.4 & 18.6 & 0.01 \\
Monogamous marriage & $76.2 \%$ & $64.4 \%$ & 0.02 \\
Single & $12.7 \%$ & $24.4 \%$ & 0.004 \\
More than 1 regular & $0.8 \%$ & $4.5 \%$ & 0.006 \\
$\quad$ partner & & & \\
Lifetime partners $\geq 4$ & $21.2 \%$ & $28.8 \%$ & 0.09 \\
HIV prevalence & $11.3 \%$ & $29.8 \%$ & $<0.001$ \\
\hline
\end{tabular}

Table 2 Prevalence of CIN and cancer in the screening and referred group

\begin{tabular}{lccc}
\hline & $\begin{array}{c}\text { Screening } \\
(548) N(\%)\end{array}$ & $\begin{array}{l}\text { Referred } \\
(105) N(\%)\end{array}$ & $\begin{array}{l}\text { Total } \\
(653) N(\%)\end{array}$ \\
\hline CIN1 & $34(6.2 \%)$ & $20(19.0 \%)$ & $54(8.3 \%)$ \\
CIN2/3 & $30(5.5 \%)$ & $24(22.9 \%)$ & $54(8.3 \%)$ \\
Inv. squamous & $1(0.2 \%)$ & $5(4.8 \%)$ & $6(0.9 \%)$ \\
\multicolumn{1}{c}{ cell carcinoma } & & & \\
\hline
\end{tabular}

services (screening group). Another 105 women were referred because of an abnormal pap smear or for clinical reasons (referred group).

The distribution of demographic characteristics and risk factors for both groups is shown in Table 1.

No significant differences in CIN detection or demographical variables were found between the group presenting for $\mathrm{V} 1$ and the group attending for V2 (data not shown).

\subsection{Detection of CIN and carcinoma}

A total of 255 biopsies were taken, on which 114 lesions were found. Table 2 shows the number of $\mathrm{CIN}$ lesions and invasive squamous cell carcinoma in the screening and the referred group. Lesions were more prevalent in the referred group. For assessment of the test qualities of the different screening methods, results from both groups are combined.

\subsection{Pap smear}

Of the 653 pap smears, 629 were "satisfactory for evaluation" (96.3\%) (Table 3), and $16.7 \%$ of the adequate smears showed LSIL or worse. All 6 cases of invasive squamous cell cancer were detected. Of the 54 CIN2/ 3 cases, 44 (81.5\%) were diagnosed on pap smear as LSIL or worse. Similarly, 27 (50.0\%) of the $54 \mathrm{CIN} 1$ cases were detected. Of the positive pap smears, $26.7 \%$ yielded a normal reference test.

\subsection{VIA}

One hundred seventy-seven VIA examinations were positive at visit $1(27.1 \%)$ (Table 3$)$. Four of the invasive squamous cell cancers were diagnosed (66.7\%), $40(74.1 \%)$ of the CIN2/3 cases, and 25 $(46.3 \%)$ of the CIN1 cases. One hundred and eight $(61.0 \%)$ positive VIA tests were normal on the reference test.

\subsection{HPV DNA PCR}

Five hundred sixteen HPV DNA PCR test results were available (Table 3 ). For hundred twenty 
Table 3 Results of screening tests compared to biopsy results

\begin{tabular}{|c|c|c|c|c|c|c|}
\hline & \multirow[t]{2}{*}{$N$} & \multicolumn{5}{|c|}{ Reference test biopsy } \\
\hline & & Normal & CIN 1 & CIN 2 & CIN 3 & Cancer \\
\hline \multicolumn{7}{|l|}{ Pap smear } \\
\hline Unsatisfactory & 24 & 23 & 1 & 0 & 0 & 0 \\
\hline Normal & 481 & 451 & 23 & 2 & 5 & 0 \\
\hline ASCUS & 43 & 37 & 3 & 2 & 1 & 0 \\
\hline LSIL & 37 & 18 & 12 & 5 & 2 & 0 \\
\hline HSIL & 50 & 7 & 14 & 5 & 23 & 1 \\
\hline Cancer & 18 & 3 & 1 & 2 & 7 & 5 \\
\hline Total & 629 & 516 & 53 & 16 & 38 & 6 \\
\hline \multicolumn{7}{|l|}{ VIA } \\
\hline Negative & 476 & 431 & 29 & 6 & 8 & 2 \\
\hline Positive & 177 & 108 & 25 & 10 & 30 & 4 \\
\hline Total & 653 & 539 & 54 & 16 & 38 & 6 \\
\hline \multicolumn{7}{|l|}{$H P V$} \\
\hline Defective $^{a}$ & 17 & 17 & 0 & 0 & 0 & 0 \\
\hline Negative & 260 & 246 & 12 & 0 & 2 & 0 \\
\hline Positive & 256 & 168 & 36 & 16 & 30 & 6 \\
\hline HPV excl. LR & 31 & 30 & 1 & 0 & 0 & 0 \\
\hline $\mathrm{HPV} \mathrm{HR}^{\mathrm{C}}$ & 193 & 108 & 34 & 16 & 29 & 6 \\
\hline HPV $X^{d}$ & 32 & 30 & 1 & 0 & 1 & 0 \\
\hline Total & 516 & 414 & 48 & 16 & 32 & 6 \\
\hline \multicolumn{7}{|l|}{ Cervicography } \\
\hline Tech defective & 15 & 11 & 1 & 1 & 2 & 0 \\
\hline $\mathrm{Neg}$ & 390 & 353 & 25 & 5 & 7 & 0 \\
\hline Atypical & 23 & 18 & 5 & 0 & 0 & 0 \\
\hline Atyp/colp & 17 & 14 & 2 & 0 & 1 & 0 \\
\hline Lgrade & 21 & 11 & 5 & 3 & 1 & 1 \\
\hline Hgrade & 51 & 17 & 10 & 5 & 16 & 3 \\
\hline Cancer & 6 & 0 & 1 & 1 & 3 & 1 \\
\hline Total & 508 & 413 & 48 & 14 & 28 & 5 \\
\hline
\end{tabular}

women, no HPV sample was taken, because of unavailability at the clinic of the PBS sampling medium, which had to be prepared freshly on a regular basis. Four HPV tests had a borderline (equivocal) result and 13 were indeterminate. These cases were excluded from the analysis.

Two hundred fifty-six (49.6\%) women had a positive HPV test, 193 (37.4\%) were infected with a HR HPV type. All invasive cancers were positive for a HR HPV type. Forty-six (95.8\%) of the CIN2/ 3 cases had a positive HPV test, 45 (93.8\%) had an HR HPV type. Thirty-six $(75.0 \%)$ of the women with CIN1 were HPV positive, $34(70.9 \%)$ were infected with an HR HPV type. One hundred sixty-eight $(65.6 \%)$ women with HPV had a normal reference test, compared to $108(56.0 \%)$ of the women with HR HPV.

\subsection{Cervicography}

A total of 523 cervicographies were taken in this group (Table 3), and fifteen images were technically defective. One hundred and thirty cervicographs are missing because of breakdown of the camera at one time. Seventy-eight of the cervicographies $(15.4 \%)$ indicated a cervical lesion. All the invasive cancer cases tested positive, $29(69.0 \%)$ of the CIN2/3 and 16 (33.3\%) CIN1. Twenty-eight (35.9\%) of the women with a positive cervicography had a normal reference test.

\subsection{Comparison of the four screening tests}

Table 4 shows estimates for the sensitivity and specificity of the four screening tests. For cases of 


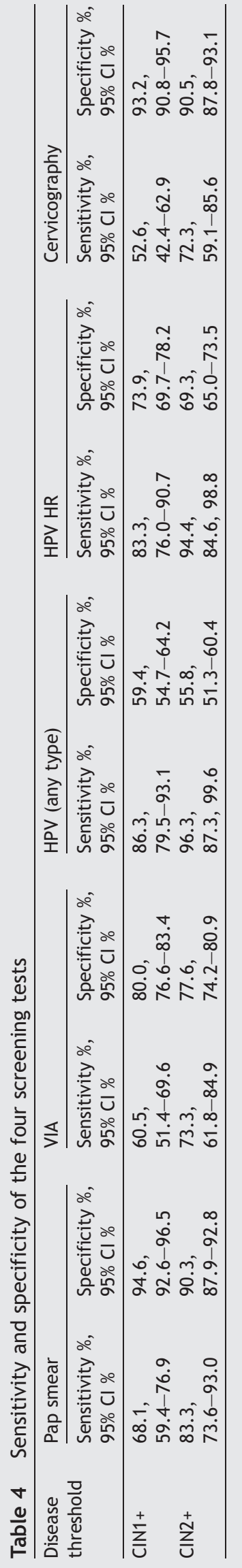

CIN2 or worse, the pap smear showed a sensitivity of $83.3 \%$ and a specificity of $90.3 \%$. The highest sensitivity (96.3\%) was found with HPV testing (any type). However, the rate of false positives was considerably higher, yielding a specificity of $55.8 \%$. Considering only HPV HR results as positive test results did not change the sensitivity significantly (94.4\%), but did increase the specificity $(69.3 \%)$. VIA scored a sensitivity and specificity of $73.3 \%$ and $77.6 \%$, respectively, and cervicography $72.3 \%$ and $90.5 \%$, respectively.

\section{Discussion}

This study was designed to compare the test qualities of alternative screening methods for the detection of $\mathrm{CIN}$ and invasive cervical cancer, which could be used in poor resource regions. This was a clinic-based population with a small proportion of referred women who contributed to the high number of (pre-)cancerous lesions.

Biopsy results were missing for 20 women with positive VIA2 but negative colposcopy, and 6 women with positive VIA2 and colposcopy suggestive of a low grade lesion. All 26 women had a negative pap smear. We kept these cases in the dataset, considering them negative on the reference test. Deleting these cases from the analysis would introduce a considerable bias towards fewer false positive VIA results.

The pap smear performed well with a sensitivity of $83.3 \%$ and a specificity of $90.3 \%$ for CIN2+. This test was performed at a referral and training center, which cautions that these results cannot be generalized for other cytology laboratories in the region. Indeed, a meta-analysis on pap test accuracy reported ranges for sensitivity and specificity of $11-99 \%$ and $14-97 \%$, respectively [7]. This author concluded that pap test accuracy was not associated with reported study characteristics or dimensions of quality. In a similar study in Zimbabwe, pap smear performed with a sensitivity and specificity of $44.3 \%$ and $90.6 \%$, respectively [8].

Most studies on HPV testing for cervical cancer screening use the Digene HPV Hybrid Capture II test, which detects 13 HR HPV types and 5 LR HPV types. Compared to our method, the Digene test had a slightly lower sensitivity and higher specificity in South Africa (88.4\% and $81.9 \%$, respectively) [9], in Costa Rica (88.4\% and $89 \%$ ) [10], in Zimbabwe (81\% and 62\%) [11], and in older women (35-45 years) in China (95\% and 85\%) [12]. The high cost and the low specificity of the test are important drawbacks. However, it has the potential 
to be used as a sole method for primary screening in women over thirty years, as HPV infection is less prevalent but more often persistent in older women [13].

Visual screening techniques have been evaluated more intensely in the last decade. The two visual screening methods, VIA and cervicography score with an almost identical sensitivity. Cervicography, however, was significantly more specific. Our study adds to the VIA test characteristics found in other studies (sensitivity and specificity for detection of $\mathrm{CIN} 2+$ disease was $71.0 \%$ and 74.3\%, respectively, in China [12], 76.6\% and 64.1\% in Zimbabwe [8], $82.6 \%$ and $86.5 \%$ in India [14], and $67.4 \%$ and $84.9 \%$ in South Africa [15]).

Two studies on cervicography provide sensitivity and specificity for CIN2+ disease of $54.5 \%$ and $97.2 \%$ [16], respectively, and $49.3 \%$ and $95.0 \%$ [6]. A study from South Africa reports sensitivity and specificity of $41.8 \%$ and $78.8 \%$, respectively, for detection of any SIL [17]. Our study reports a significantly higher sensitivity with a comparable specificity compared to the studies mentioned above.

Colposcopy and colposcopy-directed biopsy to confirm a positive test result on the primary screening test is widespread, and is recommended policy in the industrialized world. This policy is cumbersome to implement in poor-resource countries. Colposcopy facilities are costly and require highly trained medical personnel. The prospect of having one test that is sensitive enough for screening, and at the same time specific enough to direct treatment is appealing. Inevitably, a number of false-positive cases would be treated unnecessarily, but a screen- and treat approach using cryotherapy, which has relatively few side-effects, has shown to be highly acceptable in poor resource settings [18]. In addition, excision or destruction of the transformation zone are considered preventive for later development of CIN. HIV-seropositive women, however, may shed the virus more intensely in the first weeks after treatment [19], increasing infectiousness in case of unprotected sexual intercourse. During the healing period, the cervix is also more fragile and more susceptible to HIV infection. However, it is also possible that, as the cervix heals after treatment and new epithelium emerges, often with a shrunken squamo-columnar junction, the seronegative women may actually get some long term protective effect from HIV acquisition. These aspects have to be considered carefully in regions with high HIV prevalence. Pap smear and cervicography have the highest specificity and would therefore cause the least over-treatment. $\mathrm{HPV}$ testing is the least specific and the specificity of VIA is intermediate but acceptable.
The lack of cervical cancer control programs in developing countries is largely due to the cost of those programs, which have to compete for resources with other health interventions. We did not assess cost-effectiveness of the different strategies, yet VIA was shown to be a cost-saving strategy in South Africa, compared to HPV HR testing and cytology [20].

A limitation of our study is that a number of HPV tests and cervicographies are missing or noninterpretable. As this is due to logistics and/or technical shortages, bias towards the overall results for those tests is unlikely. On the other hand, it also demonstrates the difficulties with using these screening methods in poor resource settings.

In conclusion, pap smear has the highest specificity, and HPV the highest sensitivity. Cervicography and VIA are comparable and score in between. However, from the perspective of the need for a simple and widely applicable screening test for cervical (pre-)cancer in poor resource countries, our study contributes to the growing evidence of the effectiveness of VIA as a primary screening tool.

\section{Acknowledgments}

The authors gratefully acknowledge the Family Planning Association of Kenya for their continued support to the study in availing their premises and highly motivated staff, without whom the study would not have been possible.

\section{References}

[1] Miller AB, Nazeer S, Fonn S, Brandup-Lukanow A, Rehman R, Cronje $\mathrm{H}$, et al. Report on consensus conference on cervical cancer screening and management. Int J Cancer 2000; 86(3):440- 7.

[2] Miller AB, Chamberlain J, Day NE, Hakama M, Prorok PC. Report on a workshop of the UICC project on evaluation of screening for cancer. Int J Cancer 1990;46(5):761-9.

[3] Parkin DM, Pisani P, Ferlay J. Estimates of the worldwide incidence of 25 major cancers in 1990. Int J Cancer 1999; 80(6):827- 41

[4] Atlas of visual inspection of the cervix with acetic acid (VIA) [Chart]. JHPIEGO.

[5] De Vuyst H, Steyaert S, Van Renterghem L, Claeys P, Muchiri $\mathrm{L}$, Sitati $\mathrm{S}$, et al. Distribution of human papillomavirus in a family planning population in Nairobi, Kenya. Sex Transm Dis 2003;30(2):137-42.

[6] Schneider DL, Herrero R, Bratti C, Greenberg MD, Hildesheim A, Sherman ME, et al. Cervicography screening for cervical cancer among 8460 women in a high-risk population. Am J Obstet Gynecol 1999;180(2 Pt. 1):290-8. 
[7] Fahey MT, Irwig L, Macaskill P. Meta-analysis of pap test accuracy. Am J Epidemiol 1995;141(7):680-9.

[8] University of Zimbabwe, JHPIEGO. Visual inspection with acetic acid for cervical-cancer screening: test qualities in a primary-care setting. Lancet 1999;353(9156):869-73.

[9] Kuhn L, Denny L, Pollack A, Lorincz A, Richart RM, Wright TC. Human papillomavirus DNA testing for cervical cancer screening in low-resource settings. J Natl Cancer Inst 2000; 92(10):818- 25.

[10] Schiffman M, Herrero R, Hildesheim A, Sherman ME, Bratti $M$, Wacholder S, et al. HPV DNA testing in cervical cancer screening: results from women in a high-risk province of Costa Rica. JAMA 2000;283(1):87-93.

[11] Womack SD, Chirenje ZM, Blumenthal PD, Gaffikin L, McGrath JA, Chipato T, et al. Evaluation of a human papillomavirus assay in cervical screening in Zimbabwe. BJOG 2000;107(1):33-8.

[12] Belinson J, Qiao YL, Pretorius R, Zhang WH, Elson P, Li L, et al. Shanxi province cervical cancer screening study: a crosssectional comparative trial of multiple techniques to detect cervical neoplasia. Gynecol Oncol 2001;83(2): 439-44.

[13] Cuzick J, Sasieni P, Davies P, Adams J, Normand C, Frater A, et al. A systematic review of the role of human papilloma virus (HPV) testing within a cervical screening programme: summary and conclusions. Br J Cancer 2000;83(5):561-5.

[14] Sankaranarayanan R, Wesley R, Thara S, Dhakad N, Chandralekha B, Sebastian P, et al. Test characteristics of visual inspection with $4 \%$ acetic acid (VIA) and Lugol's iodine (VILI) in cervical cancer screening in Kerala, India. Int J Cancer 2003;106(3):404-8.

[15] Denny L, Kuhn L, Pollack A, Wainwright H, Wright Jr TC. Evaluation of alternative methods of cervical cancer screening for resource-poor settings [in process citation]. Cancer 2000;89(4):826- 33.

[16] De Sutter P, Coibion M, Vosse M, Hertens D, Huet F, Wesling $\mathrm{F}$, et al. A multicentre study comparing cervicography and cytology in the detection of cervical intraepithelial neoplasia. Br J Obstet Gynaecol 1998;105(6):613-20.

[17] Cronje HS, Cooreman BF, Beyer E, Bam RH, Middlecote BD, Divall PD. Screening for cervical neoplasia in a developing country utilizing cytology, cervicography and the acetic acid test. Int J Gynaecol Obstet 2001;72(2):151-7.

[18] Gaffikin L, Blumenthal PD, Emerson M, Limpaphayom K. Safety, acceptability, and feasibility of a single-visit approach to cervical-cancer prevention in rural Thailand: a demonstration project. Lancet 2003;361(9360): 814-20.

[19] Wright Jr TC, Subbarao S, Ellerbrock TV, Lennox JL, EvansStrickfaden T, Smith DG, et al. Human immunodeficiency virus 1 expression in the female genital tract in association with cervical inflammation and ulceration. Am J Obstet Gynecol 2001;184(3):279-85.

[20] Goldie SJ, Kuhn L, Denny L, Pollack A, Wright TC. Policy analysis of cervical cancer screening strategies in lowresource settings: clinical benefits and cost-effectiveness. JAMA 2001;285(24):3107-15. 